\section{RoomTetris in room committing: why the role of minimum-length-of-stay requirements should be revisited}

RoomTetris in room committing

\author{
Roberto Battiti and Mauro Brunato \\ Department of Information Engineering and Computer Science, \\ University of Trento, Trento, Italy, and \\ Filippo Battiti \\ Ciaomanager S.r.L, Trento, Italy
}

Received 28 November 2020 Revised 18February 2021 6 May 2021 15 June 2021 26 June 2021 Accepted 26 June 2021

\begin{abstract}
Purpose - This study aims to analyze how different room-committing practices affect the occupancy and profitability of hotels and it critically reviews the role of minimum-length-of-stay (MLOS) requirements given these findings.

Design/methodology/approach - The approach uses statistical analysis of simplified contexts to develop understanding, and simulations of more complex situations to confirm the relevance in realistic contexts.

Findings - The study demonstrates that proper solutions of the room-committing problem improve occupancy and profitability, in particular, for hotels working in high-season and high-occupancy situations. Smart committing algorithms diminish the role of MLOS requirements. More demand can be accepted without sacrificing late-arriving long reservations.

Originality/value - To the best of the authors' knowledge, this work, building upon a previous one cited in this paper, is the first to rigorously study the room-committing problem and to demonstrate its relevance in practical situations and its implications on MLOS rules.
\end{abstract}

Keywords Hotel management, Hotel procedures, Optimal algorithms, Room-committing problem, Simulation-based optimization, Information technology, Optimization

Paper type Research paper

\section{Introduction}

The paper (Battiti et al., 2020) introduces the "Room Committing Problem," dealing with the choice of a specific room number among the ones compatible with the sold "room type." The standard assumption is that guests are not relocated to different rooms after their check-in. This problem deals with fixing ("pinning") physical room numbers for each accepted reservation, and it has nothing to do with the "room allocation problem" considered in revenue management $(\mathrm{RM})$, in which one decides how many rooms to allocate to the

(C) Roberto Battiti, Mauro Brunato and Filippo Battiti. Published by Emerald Publishing Limited. This article is published under the Creative Commons Attribution (CC BY 4.0) licence. Anyone may reproduce, distribute, translate and create derivative works of this article (for both commercial and non-commercial purposes), subject to full attribution to the original publication and authors. The full terms of this licence maybe seen at http://creativecommons.org/licences/by/4.0/legalcode 
IJCHM

33,11

\section{8}

different "products," and different products are typically associated with different price levels. A recent analysis of hotel revenue management is Baker et al. (2020).

Traditional "joint overbooking-allocation methods" (Gallego and Van Ryzin, 1997; Zhang and Weatherford, 2017) consider a different problem of deciding about prices and number of rooms to make available for sale ("capacity allocation") in the different periods, depending on the demand forecast. Their methods estimate the opportunity cost of selling a room prematurely when it could be sold in the future at a higher price, and use dynamic programming and approximations thereof to identify pricing and capacity allocations to maximize revenue. After the optimal capacity is chosen, one is still left with a different problem of ensuring that most of the allocated capacity in the agenda can be effectively filled with reservations while avoiding guest relocations during their stay. This second problem is completely orthogonal and it exists independently of "joint overbookingallocation methods." Even if a hotel allocates all available rooms for sale and the demand is so large to potentially fill the hotel, the final achieved occupancy may be far from $100 \%$ because of the interference between short and long stays in the agenda. To avoid overbooking, some hotels close online selling on online travel agencies (OTAs) before reaching full capacity because they are not sure that additional reservations can be placed on the agenda. There can be some slots still available but there is no guarantee that an appropriate permutation of room numbers associated with reservations will create contiguous space for long stays.

It is precisely to avoid this confusion of terminology that the problem considered by us is called "Room Committing Problem" (RCP for short). The "Room Committing Problem" is often not considered worthy of attention by the management, being delegated to desk personnel, or automated software connections with different sales channels (also known as channel managers), so that a reservation is assigned a room number immediately after

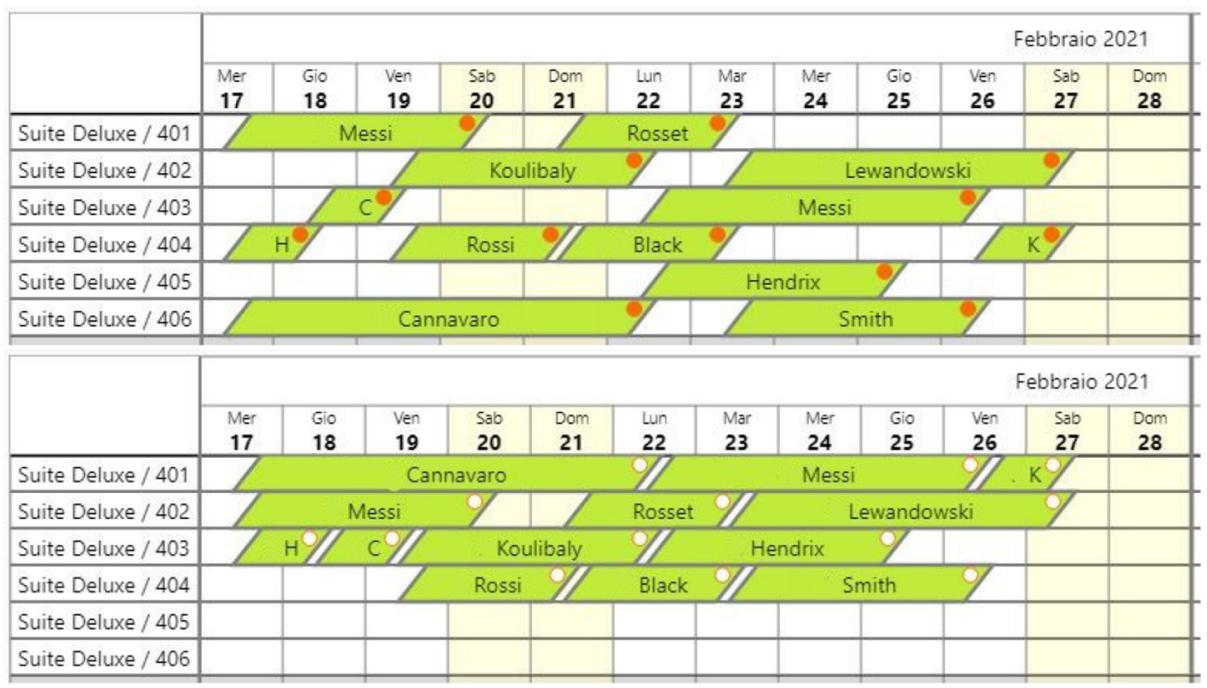

Figure 1.

Room plan (Ciaomanager PMS)
Note: Each stripe represents a reservation allocated to a specific room. Messy initial allocation (above); and optimal allocation with RoomTetris (below). Two rooms are made available to allocate even long reservations 
receiving it. When many reservations for long stays are requested by customers, this lack of strategic consideration causes inefficiencies and a sizable loss of profit because the achievable average occupancy is far from the nominal maximum availability. Indeed, a suboptimal allocation of guests into rooms creates small "bubbles" (gaps) between reservations in the room plan (the map specifying the room numbers associated with the accepted reservations). The presence of bubbles between reservations that cannot coalesce into a larger contiguous stripe to create space for long-stay reservations causes the denial of many long reservations. Denials tend to occur in high-season periods when the hotel is close to full occupancy and rooms sell for the highest prices.

A concrete case is in Figure 1, which illustrates the room plan for a period in February 2021 and the room type "Suite Deluxe." The initial situation (top figure) is messy: if a request for a long reservation in the period February 17-February 28 arrives, it is not clear if it can be allocated. The final situation after the optimal permutation executed by RoomTetris is at the bottom. Two rooms (405 and 406) are now empty and ready to accept long reservations. A red pin on a stripe means that the reservation is fixed ("pinned") to a specific room. In the normal daily operation, only the reservations with check-in today or at an earlier date are fixed, because current guests cannot be relocated, while the other reservations remain freefloating. If additional reservations arrive, the optimal situation may imply a different permutation of the free-floating ones.

In some hotels, a painful reallocation of the room numbers is executed periodically by the more expert employees in a kind of room Tetris (Demaine et al., 2003), to create larger gaps (sets of contiguous days in a specific room) and make space to accept longer reservations. The work (Battiti et al., 2020) analyzes different procedures for committing specific room numbers to reservations and defines some heuristic schemes and an optimal algorithm. The RoomTetris algorithm has the global optimality property: provided that a new reservation covers days so that at least a room is available for each day, the algorithm will identify an optimal permutation of rooms assigned to reservations to create a contiguous set of available slots. The new reservation will then be allocated to a single physical room without any guests' relocations. RoomTetris assumes that all reservations are free to be allocated to a room of the given type and that no fixed committing of room numbers is done before the check-in date. We extend the previous results in the following directions:

- Given the stochastic nature of reservation arrivals, it is not trivial to understand the effect that accepted reservations of different length of stay (LOS) have on the possible denial of future reservations. We analyze the rejection probability in simplified cases and argue that a phenomenon similar to phase transitions in physics can arise.

- We revisit the role of the traditional minimum-length-of-stay (MLOS) constraints in the light of the new algorithms and argue that, by adopting the optimal RoomTetris algorithm, the use of MLOS requirements for accepting reservations should be reconsidered. MLOS should either be eliminated or limited to cases that cannot be solved by more flexible pricing policies to encourage longer stays.

- We run extensive software simulations for realistic demand processes with seasonal effects.

In Section 2, we summarize the algorithms proposed in Battiti et al. (2020), and in Section 3, we analyze the probability that a reservation request must be rejected as a function of hotel occupancy level and reservation length. The role of minimum-stay constraints as an 
IJCHM 33,11

alternative strategy is discussed in Section 4 and compared with the optimal algorithm. Finally, experiments with more realistic demand streams are analyzed in Section 5.

\section{Summary of smart algorithms for committing rooms}

To make this paper self-contained, let us briefly summarize the algorithms introduced in Battiti et al. (2020). A reservation request can be accepted if a room is available for the entire requested period of stay (i.e., if it is free for all reservation days), and it must be rejected (or denied) otherwise. To avoid superficial complications related to nights located between two days, we assume that a hotel sells individual days. We assume that the complete state of the hotel is summarized by a RoomPlan matrix with $n_{\text {rooms }}$ rows and $n_{\text {days }}$ columns. RoomPlan [room $][d a y]$ is equal to 1 if the given room is occupied at the given day, and 0 if it is free. The list of algorithms is the following one. In all cases, if no room is available, the reservation request is rejected.

ImmediateRandom: As soon as a new request arrives, the rooms are considered in random order. The reservation is allocated to the first available room. It can be considered as a "no intelligence" benchmark.

Smarter approaches are based on the "greedy" algorithm design principle (Cormen et al., 1990), a problem-solving heuristic of making the locally optimal choice at each stage. Each reservation is allocated with some short-sighted optimization in mind, but after a reservation is fixed to a room it is never re-allocated in the future. The three considered algorithms following "greedy" principles are: ImmediateFirstFit, ImmediateMinSlack, ImmediateMaxSlack. In ImmediateFirstFit: The reservation is allocated to the first available room, considering a fixed order of consideration.

Another greedy method is motivated by avoiding the "fragmentation" of contiguous days while aiming at maintaining large gaps between reservations. Large gaps imply that there will be more contiguous days to allocate future long-duration stays. Let us define as "slack" around a reservation the number of free days immediately following or preceding it.

ImmediateMaxSlack: Among the available rooms, the reservation is placed in the room which maximizes the slack around it.

A second greedy way to reduce fragmentation of free space is to minimize the slack around a reservation, ideally placing a new reservation immediately attached to other existing reservations, again leaving other large contiguous spaces free for placing longduration stays.

ImmediateMinSlack: Among the available rooms, the reservation is placed in the room which minimizes the slack around it.

The above algorithms fix reservation requests as soon as they arrive. This early fixing is conservative and permits to answer future requests without the doubt that they cannot be fixed into the room plan. In the proposed optimal strategy, reservation requests are accepted if and only if they do not create inevitable overbooking, but they are not fixed to specific rooms until the customer checks in. If the total request of rooms is less than the total available for all days in a request, the request is accepted, otherwise, it is rejected. This criterion is necessary to accept a new reservation without creating overbooking, but not sufficient to guarantee that a suitable gap of contiguous days will be available after allocating other reservations.

The following room-committing algorithm guarantees that the above criterion is both necessary and sufficient for global optimality (Battiti et al., 2020). RoomTetris: when a reservation request is received, it is just stored in memory. Physical rooms are allocated only when the check-in day arrives. On check-in day, reservations are allocated by starting from the longest ones. The reservations are placed in the first available room. The algorithm acts 
in an online manner, without assuming knowledge of future reservations. The optimality of RoomTetris is demonstrated in Battiti et al. (2020): if there are no overbooked days, and if all reservations are free to be allocated to specific rooms of a given type, it can always fit the reservations without relocating guests during their stay.

An example of a RoomPlan for a hotel with ten rooms associated with a type is shown in Figure 2. In this simulation, a set of requests with varying LOS (from 1 to 7 days) is generated stochastically and assigned room numbers with different algorithms: Random (above), FirstFit (middle) and RoomTetris (bottom). The sequence of requests is the same for all algorithms. At the moment when the optimal algorithm reaches $100 \%$ occupancy for the ten rooms, the simulation and the generation of requests are stopped. FirstFit fills almost entirely the low-numbered rooms but it leaves many bubbles in rooms with higher numbers and causes a denial of 9.5\% reservations. Random leaves even more unoccupied spaces in all rooms and causes a denial of $15.5 \%$ reservations. Unoccupied spaces are directly related to revenues that are lost by using a suboptimal allocation and that could be salvaged by a proper permutation of rooms assigned to the various reservations.

\section{Phase transitions in rejection probability}

One of the main reasons to introduce MLOS requirements is to avoid denial of long reservations because of too many short ones already occupying the room plan. A critical issue is therefore the effect that short reservations have on the acceptance or denial of longer reservations. The presence of scattered one-day reservations will fragment the available sequences of contiguous days in the individual rooms and, therefore, reduce their average length. When a request for a long-duration reservation arrives, the probability that it must be rejected increases with the number of one-day reservations present. The available number of rooms for a specific type and the duration of stays have an impact on the final result. The objective of this section is to analyze this impact quantitatively.

In this statistical investigation, we consider the presence of one-day reservations placed randomly in the room plan and the arrival of a reservation request for $L$ days, with $L$ being greater than one. We analyze the negative effect that short (one-day) reservations already fixed to specific rooms have on the probability that the longer reservation can be accepted. In particular, we determine the occupancy level, which marks the transition between a high and low probability of acceptance.

We assume that one-day reservations are created and placed randomly and independently on the room plan, with uniform probability over the various rooms and days, so that each room on each day is occupied with a probability $p$ (equal to the occupancy ratio). We can derive the probability of rejection for a reservation of length $L$ as follows. For a specific room, the probability that a single day is free is $(1-p)$, and the probability that all days of the new reservation request are free is $(1-p)^{L}$, because of the independence assumption. The probability that the room is not available to allocate the new reservation is $1-(1-p)^{L}$. If a group of $R$ rooms is available for a given room type, the probability that all rooms are not available, and that the reservation request is rejected is $\left(1-(1-p)^{L}\right)^{R}$.

Three plots of the rejection probability for different numbers of available rooms $(1,10$ and 100) and different lengths of the new reservation request are shown in Figure 3. With one available room and a new one-day reservation, the probability is trivially a straight line (the rejection rate is equal to the occupancy level). For reservations of longer duration, the rejection rate increases rapidly, even for modest occupancy rates, because of the low probability to find all days available to fix it.
RoomTetris in room committing

4021 
IJCHM

33,11

4022
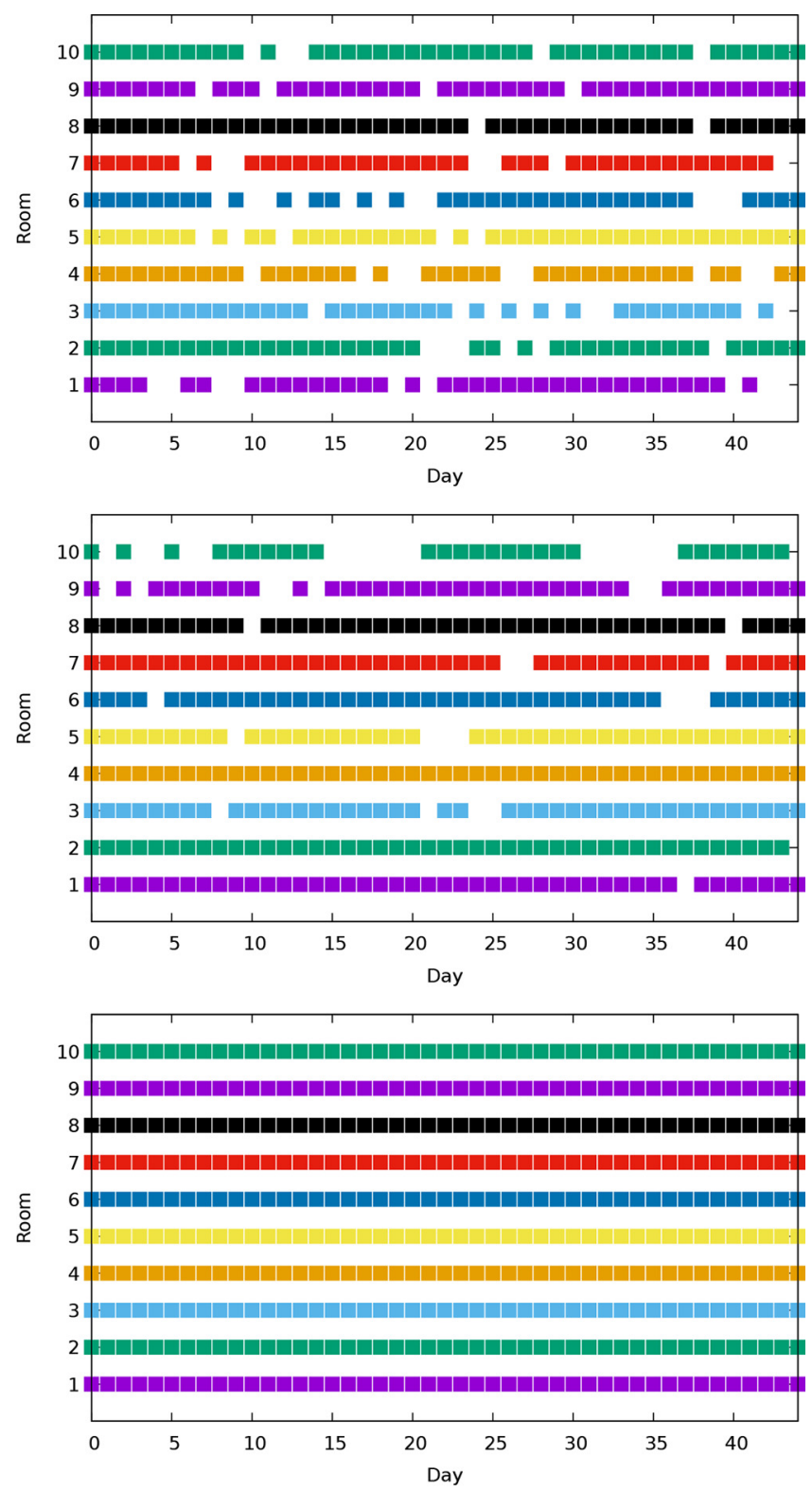

Figure 2.

Results of different room allocation strategies

Note: Random (above), FirstFit (middle) and RoomTetris (bottom). Each row describes the occupancy of a single physical room. A colored square means "room occupied" and white means "empty room." Reservation requests are randomized and accepted only if they do not cause any single-day overbooking 


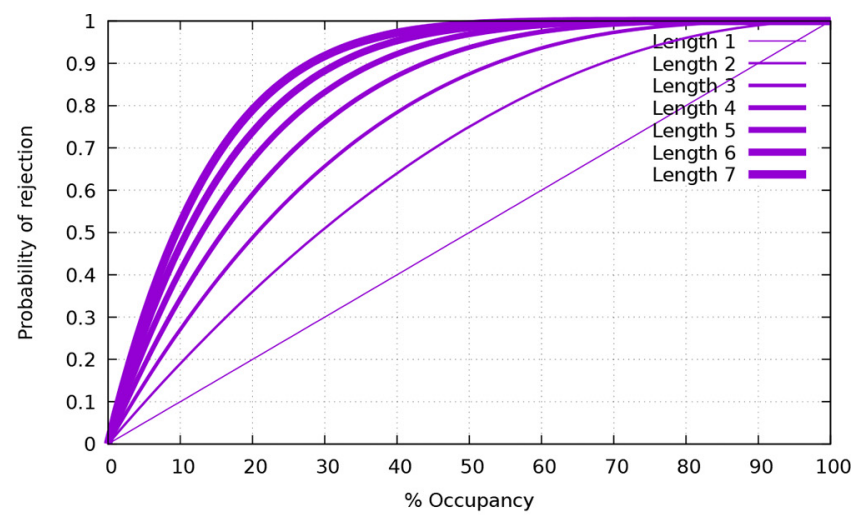

RoomTetris in

room

committing

4023
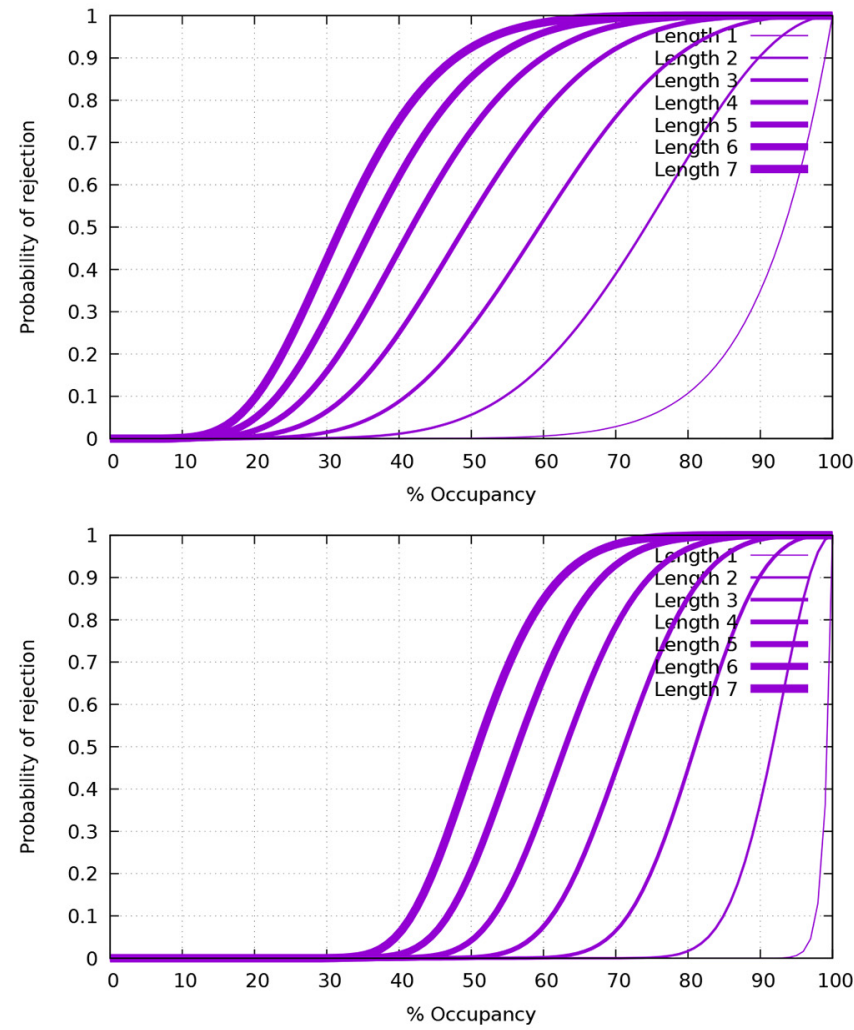

Note: The three plots are for 1 room (top), 10 rooms (middle) and 100 rooms (bottom) of a given room type. The transition to a high -rejection probability when the occupancy rate increases becomes more abrupt when the number of available rooms increases

Figure 3.

Rejection probability as a function of occupancy level 
IJCHM 33,11
The probability that an $L$-day reservation must be rejected because no room is available for the whole period tends to show a rapid transition from 0 to 1 . The transition becomes steeper and steeper the more rooms are associated with the given room type. This kind of rapid change in the possibility to solve a problem (in our case, in the possibility to find a free slot of a sufficient length) is known for many hard problems in computer science (Monasson et al., 1999) and studied with methods originating from statistical physics (Stanley, 1971).

The probability of rejection is significant even at small occupancy levels and a reasonable number of ten rooms, giving more possibilities to find an available empty period of $L$ contiguous free days. For example, at $50 \%$ occupancy, about $5 \%$ of two-day reservations must be rejected, $26 \%$ of three-day reservations, $52 \%$ of four-day reservations and up to $92 \%$ of seven-day reservations must be rejected. This result has a dramatic impact on profitability; even if the hotel is $50 \%$ empty (on average), the large majority of one-week reservations must be rejected. Even if 100 rooms are associated with a given room type (a situation which can be found only in the largest hotels), a careless fixing of 1-day reservations will lead to a rejection of about half 1 -week requests at a 50\% occupancy level.

Although the assumptions of random allocation considered in this analysis represent an extreme case, the situation in which rooms are allocated without smart algorithms and room types are not defined strategically are frequent in many hotels. The case is particularly dramatic for hospitality businesses that do not use types but sell individual rooms. In their case, this obstructive effect caused by short stays is significant even at modest occupancy rates (top plot of Figure 3).

\section{Revisiting the role of minimum-stay constraints}

The statistical analysis in the previous section was a simplified scenario. We now consider a traditional method to maintain space in the room plan to be able to accept longer reservations. The technique is based on LOS constraints. LOS was studied in hospitality management in the context of using LOS controls to optimize revenues (Jain and Bowman, 2005; Pekgün et al., 2013; Quain et al., 1999; Wilson et al., 2015), traveler satisfaction, expenditure and accommodation selection (Barros and Machado, 2010; Salmasi et al., 2012). LOS controls have been used to "shift demand from sold-out periods to slack periods" (Quain et al. 1999), and to make customers stay at your hotel for a longer period than they want. For example, New Year's Eve is a hot day with many requests, but selling it as a single-day prejudices the possibility to sell a longer-duration period.

Weatherford (1995) states that new, sophisticated LOS heuristic controls can allow hotels to reap benefits as high as about $3 \%$ of revenues, depending on the characteristics of the property. Legal implications are discussed in Wilson (2001): the MLOS controls appear to violate state case law and state statutes that require a hotel to provide a room when rooms are available. By setting extremely high daily rack rates for days of the year where expected demand is high and where the hotel wants to discourage one-night stays, the hotel can still offer a room to all who desire to stay for just one night. The hotel can set a rate high enough to offset the revenue generated by a longer stay and does not have to turn away a guest when there is availability. Empirical studies of the relationship between prices and LOS are analyzed in Riasi et al. (2017), which discovers in their context that longer stays are correlated with higher daily prices, contrary to the belief that quantity discounts should always be applied. Recently, Barros and Machado (2010) measure a declining average in the tourism LOS, and people tend to travel more often but with shorter vacations.

In this section, we study the degree to which LOS controls can be omitted, or greatly reduced, through a smarter placement of rooms. If one does not consider very special days (like New Year's Eve) and assumes a random mixture of short- and long-stay reservation 
requests, short-stay reservations occurring early make long stays more and more difficult to fit. Having a large required MLOS at the beginning of the reservation window is a way to encourage the placement of long reservations before short ones. Unfortunately, the competition is only one click away in internet time, and rejecting a customer request implies losing the related profit, with a high probability. Few customers have the patience to wait and come back later to see if the minimum-stay requirements have been canceled. In addition, removing the MLOS later in the booking window because of lack of demand is risky because people could cancel and rebook without the MLOS, or it may cause negative customer reactions for those who accepted the MLOS.

If the hotel manager is not careful, MLOS requirements can lead to a situation in which no long-term reservation (longer than or equal to the MLOS) is possible, although the hotel is not fully booked. Some days are still available but the remaining slots are just too short to fit long reservations and will never be sold. In the following, we study maximum occupancy reachable with a mixture of long and short random reservations. The unconstrained demand is generated to be equal to the maximum occupancy, but with randomized overbooking possible because of the "balls into bins" phenomenon explained in Section 4.1.

Let $p_{1}$ be the probability of generating one-day reservations, so that $1-p_{1}$ is the probability to generate seven-day reservations. The experiments study the effects on the final occupancy rate of varying probabilities for short reservations, for different room allocation algorithms. Figure 4 shows the results for a randomized committing (in a firstcome-first-fixed manner, provided that no overbooking is created). Figure 5 shows the results obtained with a strict MLOS requirement of two. Figure 6 shows the results obtained by RoomTetris in the same conditions, without any MLOS requirement.

There is only a common result between the two contexts of Figures 4 and 6, the case corresponding to having only one-day reservation requests (the rightmost points in the plots). For example, if only one room is available, the reached occupancy rate is $63.17 \%$ in both cases. The only rejected requests are in both cases those corresponding to overbooked days. At first, given that 365 randomized reservation requests are generated during a year,

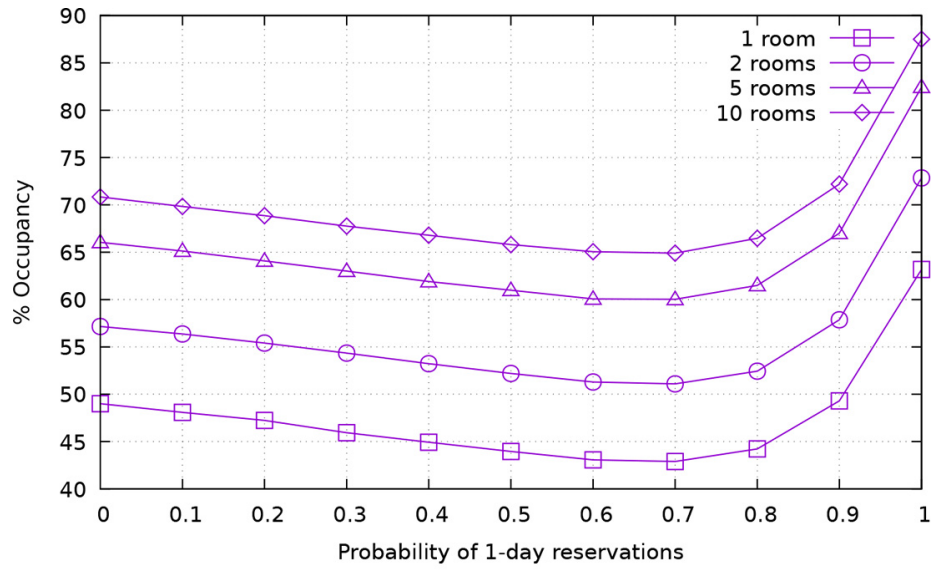

Note: Occupancy level for different number of available rooms of the same type (from one to ten), as a function of the frequency of one-day reservation requests (randomized demand equal to total capacity)
RoomTetris in room committing

4025
Figure 4 .

Randomized placement 
IJCHM

33,11

\section{6}

\section{Figure 5.}

Randomized placement with strict minimum-stay requirement

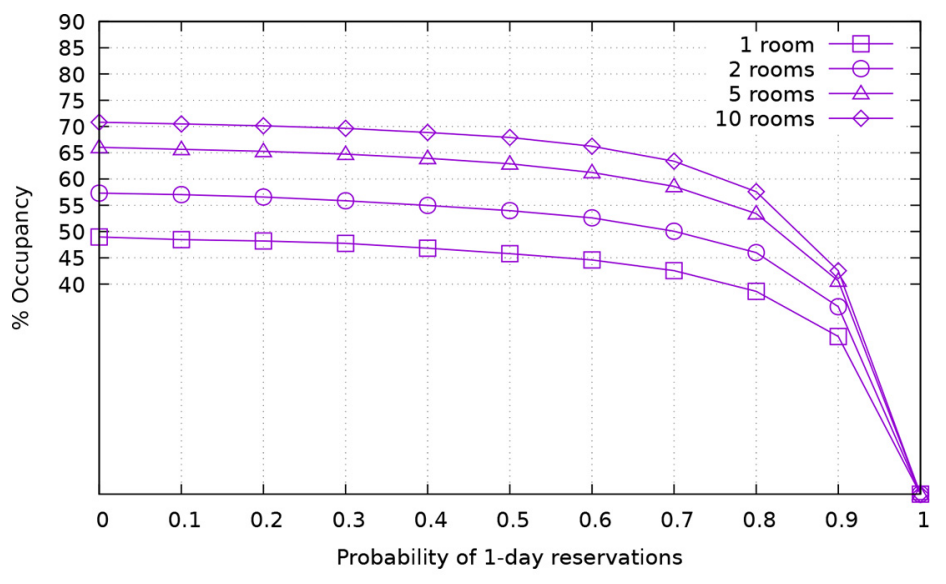

Note: Occupancy level for different number of available rooms of the same type (from one to ten), as a function of the frequency of one-day reservation requests (randomized demand equal to total capacity)

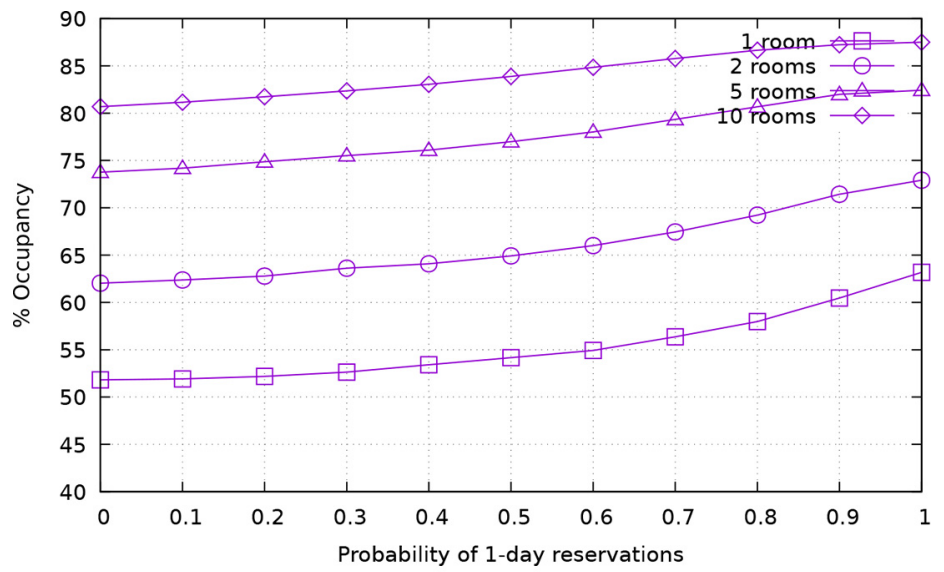

Note: Occupancy level for different number of available rooms of the same type (from one to ten), as a function of the frequency of one-day reservation requests (randomized demand equal to total capacity)
Figure 6. RoomTetris placement

the occupancy level of $63.17 \%$ looks surprisingly low. The explanation is related to the "balls into bins" phenomenon explained in Section 4.1.

Apart from this very particular case, there is a qualitative difference between the algorithms. In the random placement of Figure 4, one notices a decrease in occupancy level when the probability of a one-day reservation increases up to about 0.7. In this case, the presence of short-duration stays reduces the probability to accept longer stays. This is a strong motivation for having MLOS in the hotel business rules. The situation is particularly 
dramatic for a single room. One starts from an occupation level of $49 \%$ (because of the random placement, some seven-day requests share some days and are therefore in overbooking), drops to a level of $42.9 \%$ for a 0.7 probability of one-day reservations, and then increases again with a predominance of one-day reservations. The more rooms are available, the larger is the final occupancy level: because of statistics, there are more rooms to try before abandoning a reservation request.

If a strict MLOS of two is adopted for all reservations (only requests for more days are accepted), the result is illustrated in Figure 5. For small probabilities of one-day reservations (e.g. $p_{1}=0.1$ or $p_{1}=0.2$ ), the occupancy tends to remain much more stable than without MLOS requirements, and the allocation of the seven-day reservations is not spoiled by the presence of one-day ones. When the fraction of one-day reservations increases, the occupancy decreases rapidly because all one-day reservations are lost given the MLOS. Of course, it is expected that an intelligent hotel owner will relax the minimum stay requirement when the check-in date is getting closer, but the precise timing is complex and requires precise estimation of the demand. For example, if most requests for short reservations occur before relaxing the constraint, they will be lost forever. Let us underline that this late relaxation of MLOS could create cancellations (and rebooking without the MLOS) or negative reactions by customers who accepted the MLOS. With the growing diffusions of OTAs, price comparison sites and public daily prices on hotel booking engines, the information asymmetry between hoteliers and customers is rapidly decreasing and business practices that made sense years ago should be strongly revised. The current usage of MLOS is one of the practices which needs a strong revision. Long-term forecasts (Weatherford and Kimes, 2003) are indispensable for rigid MLOS rules, but the difficulties of accurate long-term forecasts are increasing given the rapid changes in customer preferences and the highly dynamic competitive context. In this rigid-MLOS rule without late relaxation, after the occupancy level is high and the presence of many short holes in the agenda implies that MLOS cannot be satisfied anymore, additional reservation requests will be denied. The remaining "fragmented" inventory will be lost forever, together with the associated revenue.

The situation is qualitatively different in Figure 6: in this case, the occupancy level keeps increasing when there are more one-day reservations. If a room is available, the priority given by the RoomTetris strategy to long reservations favors a better utilization of the single room. If more rooms are present, the performance rapidly improves to reach a level between $80.68 \%\left(p_{1}=0\right)$ and $87.5 \%\left(p_{1}=1\right)$.

The above results demonstrate that the RoomTetris placement of rooms is a recommended alternative to LOS controls to reach a higher occupancy level and a higher profit. Of course, a second motivation for MLOS requirements can be related to the cost structure. For example, if one is renting apartments with a fixed price per day and a high variable cost per reservation for a complete cleaning, the marginal profit may well become negative for a very short reservation. A possible alternative is to differentiate the cost according to the duration, either with an explicit cleaning fee or, depending on marketing and communication, with a decreasing cost per day for longer reservations. In both cases, the choice will be up to the customers: if they are prepared to pay more for short stays, they should be allowed to do it. Hotels will increase potential revenues if customers accept variable prices.

\subsection{Balls and bins: stochastic arrivals reduce expected occupancy}

In the previous experimental setting, we assumed that the total number of requested days is equal to the total available inventory and ended up with $12 \%$ excess demand for ten available rooms, caused by overbooked days. Although puzzling, this result is easily 
IJCHM

33,11

4028

explained by the "balls into bins" problem. Even if the total request of rooms is equal to the total available inventory, statistical fluctuations create a variation of demand in the individual days, leading to under-booked and overbooked days.

The "balls into bins" problem in probability theory (Kolchin and Sevast, 1978) involves $m$ balls and n boxes (or "bins"). Each time, a single ball is placed into one of the bins, in a stochastic manner with uniform probability for all bins. After all the balls are in the bins, one looks at the number of balls in each bin - defined as the load on the bin - and asks a variety of questions. In our case, a ball is a single one-day reservation and a bin is a single day.

Given $n$ balls and $n$ bins, if one throws balls into bins sequentially uniformly at random, the probability for a ball $i$ to fall into a given bin is exactly $P$ (ball $i$ falls into bin $i$ ) $=1 / n$. If $X$ denotes the number of empty bins, then one can demonstrate that: $E[X]=n(1-1 / n)^{n}$ similar to $n /$ e for large $n$ values. The fraction of empty bins is equal to $1 / \mathrm{e} \approx 0.3678$. In the case of one-day reservations occurring randomly with the same probability for all days, $1 / e$ is also the approximate ratio of rejected reservations. They ended up in occupied days and therefore had to be rejected. In this case, the estimated occupancy is $1-0.3678 \approx 0.6322$, in close agreement with the $63.17 \%$ occupancy measured in the simulation in the previous section for the case of one-day reservations and one room.

To demonstrate the above results, one can use indicator variables. Let $Y$ be the number of empty bins. One wants to calculate the expected value $E[Y]$. If one defines the indicator variables $X_{i}$ so that $X_{i}$ is 1 if bin $i$ is empty and 0 otherwise, one obtains:

$$
Y=X_{1}+X_{2}+\ldots+X_{n}
$$

By linearity of expectation,

$$
E[Y]=E\left[X_{1}\right]+E\left[X_{2}\right]+\ldots+E\left[X_{n}\right]
$$

So now the problem reduces to calculating the $E\left[X_{i}\right]$ for each $i$. But this is fairly easy, as $E\left[X_{i}\right]=1 P\left(X_{i}=1\right)+0 P\left(X_{i}=0\right)=P\left(X_{i}=1\right)=P($ bin $i$ is empty $)=(1-1 / n)^{m}$, where the last equality is because balls $1,2, \ldots, m$ must all go in a bin other than $i$, each with probability $(1-1 / n)$.

This simplified context reminds us that, in general, the stochasticity of demand is bound to reduce the achievable occupancy. Even if the expected demand for each day could make the owner think that the hotel will be fully booked, its stochasticity can greatly reduce the experimented occupancy. This conclusion holds unless the hotel finds ways to convince customers to shift reservations to nearby days which are still available when the requests arrive, for example, for tourists without rigid constraints.

\section{Simulating realistic demand processes}

This part is concerned with simulating more realistic situations, to assess whether the superiority of RoomTetris is confirmed also for more complex scenarios. Experimenting with real hotels is notoriously difficult because it involves assuring repeatability - while the overall context is always changing - and because experiments tend to interfere with daily activities. On the other hand, a growing fraction of experimental science is currently based on realistic software simulators which are extremely useful also for hospitality research. Tunable simulators with stochastic demand generators can be adapted to different scenarios and are a promising alternative to in vivo experiments. The reproducibility of results is guaranteed, and a sound statistical analysis can be based on analyzing hundreds or thousands of simulations, with different seeds for the random number generators. 
In general, an optimizing modeling approach to hospitality research has the advantage to address the increasing levels of management complexity that characterizes modern hotels, without being limited by unrealistic assumptions. The interplay of various choices and strategies can be modeled by running simulations, and optimal or at least improving settings can be obtained through simulation-based optimization like it is routinely done in many modern engineering areas (Gosavi, 2015). In an era of big data, one can calculate probabilities in real-life scenarios with good accuracy, and the approach becomes practical. The final expected results on financial key performance indicators can be easily predicted as soon as the macroscopic characteristics of the demand (seasonality, distribution of LOS and expected occupation levels) and the pricing strategy are fed to software simulators like the one developed by our research team.

For example, a computer-simulated hotel-reservation and revenue-management system was used in Choi and Kimes (2002) to test electronic distribution channels' effect on hotel revenue management. Zakhary et al. (2011) uses Monte Carlo simulation for forecasting hotel arrivals and occupancy. A simulation-based approach for the overbooking problem is adopted in Fouad et al. (2014). Hotel simulation as a decision-making tool for learning and promoting users' confidence is considered in Ampountolas et al. (2019). A flexible discreteevent simulator for the hotel reservation process and an experimental methodology which carefully separates a "training" phase from the final "validation" phase is studied in Brunato and Battiti (2020).

A more realistic demand distribution has been implemented by simulating seasonal variability of demand. The two time-dependent parameters are the expected number of reservations per day $(R)$ and the expected LOS $(L)$, which have been modulated to describe the typical bimodal distribution of a mountain town, with a winter and a summer season, with shorter, business-like stays throughout the year. The duration of the simulated period is one year, and the number of rooms is 50 . The parameters for all months, represented by a couple of values $(R, L)$ are: January: $(15,2)$; February: (20, 4); March: $(25,4)$; April: $(10,2)$; May: (5, 1.5); June: (10, 2); July: (15, 4); August: $(25,4)$; September: $(15,2)$; October: $(5,1.5)$; November: $(5,1.5)$; and December: $(15,2)$.

Reservations have been generated by a Poisson process whose number of events per day is regulated as explained before. Overbooking is not accepted: a reservation request is accepted only if does not generate any single-day (inevitable) overbooking. This criterion also agrees with the last room availability condition with OTAs. The hotel communicates to the OTA the number of rooms available for each type and each future day (in the booking horizon), and OTAs accept reservations if and only if each day in the reservation has availability for sale of at least one.

As for the LOS, two different distributions have been tested. In the first case, only two values (one day and seven days) were allowed, to simulate the behavior of a hotel that encourages weekly accommodations, but also accepts short filler reservations from business travelers; in this case, the probability of the two outcomes is adjusted to obtain the expected value of $L$ given above. As an alternative, the LOS has been allowed to take any positive integer value by rounding (to the ceiling) the output of an exponential distribution generator whose mean value varies according to given $L$ values.

Table 1 collects the results of the two realistic simulations, for a mixture of one- and seven-day reservations (case A) and a more complex mixture of durations (case B). The standard error of the mean (SEM) is reported for the mean rejection rate. Given that 1,000 different tests are executed, this error is already very low.

The results in the cases are qualitatively similar. In case A of a simple mixture, random loses about $12 \%$ of the reservations, the smarter committing algorithms about $3 \%$, while all reservations are fixed with no loss by RoomTetris. In case B of a more complex mixture, the
RoomTetris in room committing

4029 
IJCHM

33,11

4030

final occupancy by all techniques improves. This result can be rationalized: if one starts from a set of reservations of different lengths $(1,2,3 \ldots 7)$, there are statistically more ways to fill the remaining slots, leading to a higher final occupancy rate. The simple mixture of oneand seven-day reservations considered for many experiments in this paper should therefore be considered more challenging for more diverse mixtures of reservations.

For a context with strong seasonality like the one considered, given that smart roomcommitting algorithms tend to release additional occupancy in the high-season and highprice periods, the expected percent increase of revenue will tend to be significantly larger than the expected percent increase of occupancy, depending on the price variability between the different periods.

\section{Discussion and conclusions}

It is widely recognized that applications of information technology and quantitative methods in tourism and hospitality deliver a growing competitive advantage (Bilgihan et al., 2011; Baggio and Klobas, 2017; Law et al., 2019). Inefficiencies in the UK hotel industry are investigated in Tan and Despotis (2021), while factors influencing profitability in the Italian hospitality industry are studied in Menicucci (2018). Sainaghi et al. (2013) argues that "the information and communication variables showed the great potential which technology management may reveal, above all, in monitoring the various perspectives, in feeding new performance indices and in managing efficiency and productivity in management processes."

In this paper, we focus on an aspect that has been almost neglected by research in hospitality: the process by which specific physical rooms are fixed to reservations before the guests' check-in. The topic shows an interesting mix of information technology, internal and external management processes, statistics and algorithms related to optimal permutations. The relevance (Sainaghi et al., 2019) of the "room committing problem" is demonstrated by the fact that the seemingly irrelevant decisions about assigning room numbers to reservations can have a dramatic impact on profitability, especially in high-season periods, when the hotel is near to full occupancy and prices are higher.

\subsection{Conclusions}

This paper started from the previous work (Battiti et al., 2020) and studied the interplay between optimal room-committing strategies and MLOS requirements. We analyzed the

Table 1.

Results of roomcommitting algorithms on realistic demand curves

\begin{tabular}{lcccc}
\hline Cases and algorithms & Rejection rate (\%) & SEM & Rejected days & Occupancy (\%) \\
\hline Case A & & & & \\
Random & 12.07 & 0.11 & 404.04 & 80.67 \\
FirstFit & 2.74 & 0.05 & 91.84 & 89.22 \\
MinSlack & 2.74 & 0.05 & 91.84 & 89.22 \\
MaxSlack & 3.19 & 0.06 & 106.89 & 88.81 \\
RoomTetris & 0 & 0 & 0 & 91.74 \\
Case B & & & & \\
Random & 11.07 & 0.08 & 382.39 & 84.19 \\
FirstFit & 4.46 & 0.06 & 154.21 & 90.44 \\
MinSlack & 4.45 & 0.06 & 153.80 & 90.45 \\
MaxSlack & 4.71 & 0.06 & 162.94 & 90.20 \\
RoomTetris & 0 & 0 & 0 & 94.67 \\
\hline
\end{tabular}


effect of different room-committing practices on the occupancy and profitability of hotels, and we critically reviewed the role of MLOS.

We demonstrated that intelligent solutions of the room-committing problem improve occupancy and profitability, also in realistic situations. Smart committing algorithms diminish the role of MLOS requirements, so much that they can be omitted in most situations. More demand can be accepted flexibly without sacrificing late-arriving long reservations.

Customers on the Web are used to the immediate gratification provided by a seamless user experience and are easily diverted to competing hotels if their requests are not answered immediately. The times of customers patiently waiting for a confirmation or stoically adapting to rigid rules such as MLOS or specific arrival and departure dates (like one week from Saturday to Saturday) are gone for good. Given the high practical relevance of decisions related to MLOS requirements, our findings will catalyze a reconsideration of standard business practices in hotel management, with significant implications on profits.

\subsection{Theoretical implications}

The approach used statistical analysis of simplified contexts to develop understanding, and simulations of more complex situations to confirm the relevance in realistic contexts. Some areas in hospitality research can be profitably enriched by methods of experimental science. In vivo experiments are costly and can interfere too much with the hotel's daily operations, but simulation-based research like that of our paper is a pragmatic surrogate. Softwarebased simulators can become "hospitality wind tunnels" to learn more about complex processes in the area.

Tunable software simulators can be rapidly adapted to specific hotel and demand characteristics to evaluate different decisions. Estimated averages and error bars (related to the risk) can be obtained by repeating many stochastic simulations.

\subsection{Practical implications}

Management should spotlight internal procedures and automated methods to allocate guests to rooms. The processes studied in our work are simple to adopt via automated software systems. The revenue increase can be around 3\%-10\% for hotels operating close to full occupancy in high season and having a mixture of reservations of different lengths of stay. Depending on the current hotel's net profit margin, this impact can be extremely relevant. Well-organized hotels are already using some form of "smart" room allocation mechanisms. Our research adds the scientific rigor of measuring the effects on financial performance in more complex and realistic contexts via tunable software simulators.

The findings are particularly relevant also for family run businesses, which are slower to adopt information technology and software (Kilic and Okumus, 2005). The availability of sophisticated services in the cloud at affordable prices will make them reap productivity levels like those of the larger and better-organized hotels.

Methods and internal processes which fix rooms prematurely and rigidly tend to deteriorate the optimal achievable occupancy. Given the interference between short- and long-duration stays, short stays scattered on the room plan prejudice accepting future longduration reservations.

The difficulties in finding contiguous slots in the room plan increase when the hotel is almost full. Therefore, the percent difference in occupancy levels reachable by smarter allocation algorithms produces amplified revenues because high-request and high-season periods are related to higher prices.
RoomTetris in room committing 
IJCHM

33,11

Adding MLOS requirements is a traditional way to avoid this negative effect of shortduration stays, but the price to pay can be high: renouncing many short reservations tends to decrease profit because the average customer is not patient, does not wait for requirements to be relaxed and often switches to a competing and better-organized hotel. In addition, late relaxation of MLOS is risky for the hotel's reputation. Earlybird customers should be treated with respect and fairness.

Provided that the optimal RoomTetris algorithm is adopted, hotel managers should seriously reconsider the use of MLOS requirements, limiting them to cases that cannot be solved by more flexible pricing giving more choice to the customers. MLOS may not be needed as frequently when using RoomTetris, particularly when forecasts are inaccurate and the decision to implement an MLOS is tricky. One complication is that the unconstrained demand is unknown and difficult to estimate from the "censored" observed demand.

One needs to distinguish between two kinds of overbooking. Inevitable overbooking occurs if some days in a reservation are completely sold out. Avoidable overbooking arises because of the suboptimal assignment of reservations to rooms. OTAs request from the hotels only availabilities for the different days (not detailed room plans) and some hotels are forced to close online selling prematurely when they are almost sold out to ward off avoidable overbookings. They are afraid to receive reservations by OTAs which cannot be assigned to a single physical room and which cause painful monetary and reputation penalties. The RoomTetris algorithm makes this premature closure unnecessary so that the hotels will sell all available slots and eliminate the headaches related to overbooking.

In addition to marketing and customer segmentation, the strategic choice of room types should also consider the effects on room allocation. Statistics will help in allocating rooms because of some forms of the "law of large numbers" (Dekking et al., 2005). When the numbers of rooms for each type become exceedingly small, the possibility of permuting rooms decreases, and the negative effects of sub-optimal allocations are magnified.

\subsection{Limitations and future research}

A limitation of the research is the considered context of reservations, which can be fixed to arbitrary rooms in each "room type" set. If a subset of rooms need to be fixed before check-in day (e.g. because the customer is allowed to specify the preferred room number), more complex heuristics need to be developed. A promising avenue for further research is on the interaction between traditional RM methods and room-committing strategies. As explained, the two problems are different, but choices in both areas can interact. As an example, the opportunity cost of allowing a customer to choose room numbers should be evaluated so that different reservation rules can be designed with different pricing strategies.

\section{References}

Ampountolas, A., Shaw, G. and James, S. (2019), "Active learning to improve self-confidence and decision-making skills through the use of hotel simulation", Journal of Hospitality and Tourism Education, Vol. 31 No. 3, pp. 125-138.

Baggio, R. and Klobas, J. (2017), Quantitative Methods in Tourism: A Handbook, Channel view publications Bristol.

Baker, T., Eziz, A. and Harrington, R.J. (2020), "Hotel revenue management for the transient segment: taxonomy-based research" International Journal of Contemporary Hospitality Management, Vol. 32 No. 1, pp. 108-125. 
Barros, C.P. and Machado, L.P. (2010), "The length of stay in tourism", Annals of Tourism Research, Vol. 37 No. 3, pp. 692-706.

Battiti, R., Brunato, M. and Battiti, F. (2020), "RoomTetris: an optimal procedure for committing rooms to reservations in hotels", Journal of Hospitality and Tourism Technology, Vol. 11 No. 4, pp. 589-602.

Bilgihan, A., Okumus, F. and Kwun, D.J.-W. (2011), "Information technology applications and competitive advantage in hotel companies", Journal of Hospitality and Tourism Technology, Vol. 2 No. 2.

Brunato, M. and Battiti, R. (2020), "Combining intelligent heuristics with simulators in hotel revenue management" Annals of Mathematics and Artificial Intelligence, Vol. 88 No. 1-3, pp. 71-90.

Choi, S. and Kimes, S.E. (2002), "Electronic distribution channelsstics witon hotel revenue management", The Cornell Hotel and Restaurant Administration Quarterly, Vol. 43 No. 3, pp. 23-31.

Cormen, T.H., Leiserson, C.E. and Rivest, R.L. (1990), Introduction to Algorithms, McGraw-Hill, New York, NY.

Dekking, F.M., Kraaikamp, C., Lopuhaä, H.P. and Meester, L.E. (2005), A Modern Introduction to Probability and Statistics: Understanding Why and How, Springer Science and Business Media, New York, NY.

Demaine, E.D., Hohenberger, S. and Liben-Nowell, D. (2003), "Tetris is hard, even to approximate", in 'ntris is hard, even to aand Combinatorics Conference', Springer, New York, NY, pp. 351-363.

Fouad, A.M., Atiya, A.F., Saleh, M. and Bayoumi, A.E.-M.M. (2014), "A simulation-based overbooking approach for hotel revenue management", 10th international computer engineering conference (ICENCO) a, IEEE, pp. 61-69.

Gallego, G. and Van Ryzin, G. (1997), "A multiproduct dynamic pricing problem and its applications to network yield management” Operations Research, Vol. 45 No. 1, pp. $24-41$.

Gosavi, A. (2015), "Simulation-based optimization: an overview", in 'nmulation-based optimization: Springer, New York, NY, pp. 29-35.

Jain, S. and Bowman, H.B. (2005), "Measuring the gain attributable to revenue management", Journal of Revenue and Pricing Management, Vol. 4 No. 1, pp. 83-94.

Kilic, H. and Okumus, F. (2005), "Factors influencing productivity in small island hotels", International Journal of Contemporary Hospitality Management, Vol. 17 No. 4.

Kolchin, V.F. and Sevast, B.A. (1978), 8Random Allocations, ocaVH Winston and Sons, Washington, $D C$.

Law, R., Leung, D. and Chan, I.C.C. (2019), "Progression and development of information and communication technology research in hospitality and tourism", International Journal of Contemporary Hospitality Management, Vol. 32 No. 2, pp. 511-534.

Menicucci, E. (2018), "The influence of firm characteristics on profitability", International Journal of Contemporary Hospitality Management, Vol. 30 No. 8, pp. 2845-2868.

Monasson, R., Zecchina, R., Kirkpatrick, S., Selman, B. and Troyansky, L. (1999), "Determining computational complexity from characteristic "phase transitions", Nature, Vol. 400 No. 6740, p. 133.

Pekgün, P., Menich, R.P., Acharya, S., Finch, P.G., Deschamps, F., Mallery, K., Sistine, J.V., Christianson, K. and Fuller, J. (2013), "Carlson Rezidor Hotel group maximizes revenue through improved demand management and price optimization”, Interfaces, Vol. 43 No. 1, pp. 21-36.

Quain, W.I., Sansbury, M. and Quinn, D. (1999), "Revenue enhancement, part 3: picking low-hanging fruit-a simple approach yield management", Cornell Hotel and Restaurant Administration Quarterly, Vol. 40 No. 2, pp.76-83.
RoomTetris in room committing

4033 
IJCHM

33,11
Riasi, A., Schwartz, Z., Liu, X. and Li, S. (2017), "Revenue management and length-of-stay-based room pricing”, Cornell Hospitality Quarterly, Vol. 58 No. 4, pp. 393-399.

Sainaghi, R., Phillips, P. and Corti, V. (2013), "Measuring hotel performance: using a balanced scorecard perspectives' approach”, International Journal of Hospitality Management, Vol. 34, pp. 150 -159.

Sainaghi, R., Phillips, P., Baggio, R. and Mauri, A. (2019), "Hotel performance: rigor and relevant research topics”, International Journal of Hospitality Management, Vol. 78, pp. 13-26.

Salmasi, L., Celidoni, M. and Procidano, I. (2012), "Length of stay: price and income semi-elasticities at different destinations in Italy", International Journal of Tourism Research, Vol. 14 No. 6, pp. 515-530.

Stanley, H.E. (1971), Phase Transitions and Critical Phenomena, Clarendon Press, Oxford.

Tan, Y. and Despotis, D. (2021), "Investigation of efficiency in the UK hotel industry: a network data envelopment analysis approach", International Journal of Contemporary Hospitality Management, Vol. 33 No. 3, pp. 1080-1104.

Weatherford, L.R. (1995), "Length of stay heuristics: do they really make a difference?", Cornell Hotel and Restaurant Administration Quarterly, Vol. 36 No. 6, pp. 70-79.

Weatherford, L.R. and Kimes, S.E. (2003), "A comparison of forecasting methods for hotel revenue management", International Journal of Forecasting, Vol. 19 No. 3, pp. 401-415.

Wilson, R. (2001), "Minimum length-of-stay requirements as part of hotel revenue management systems: are they legal?", The Journal of Hospitality Financial Management, Vol. 9 No. 1, pp. 45-54.

Wilson, R.H., Enghagen, L.K. and Lee, M. (2015), "Dynamic pricing and minimum length of stay controls as a hotel management practice: are there customer perception, ethical, and legal questions?", The Journal of Hospitality Financial Management, Vol. 23 No. 2, pp. 107-123.

Zakhary, A., Atiya, A.F., El-Shishiny, H. and Gayar, N.E. (2011), "Forecasting hotel arrivals and occupancy using Monte Carlo simulation”, Journal of Revenue and Pricing Management, Vol. 10 No. 4, pp. 344-366.

Zhang, D. and Weatherford, L. (2017), "Dynamic pricing for network revenue management: a new approach and application in the hotel industry", INFORMS Journal on Computing, Vol. 29 No. 1, pp. 18-35.

\section{Corresponding author}

Roberto Battiti can be contacted at: roberto.battiti@unitn.it

For instructions on how to order reprints of this article, please visit our website: 